\title{
Design of AR Inspection System for Protection Equipment of Intelligent Substation
}

\author{
Yuming Liu ${ }^{1,2 *}$, Shucheng Yang ${ }^{1}$, Wenyan Guan ${ }^{1}$, Zhe Han $^{1}$, Yangdong Qian ${ }^{3}$ \\ ${ }^{1}$ National Engineering Research Center of System Simulation Technical Application in Economy, Beijing,100854, People's Republic of \\ China \\ ${ }^{2}$ Dept. Computer Science and Technology, Tsinghua University, Beijing, 100084, People's Republic of China; \\ ${ }^{3}$ Aerospace Yinshan Electric Co., Ltd, Zhuhai, Guangdong, 519040, People's Republic of China
}

\begin{abstract}
According to the requirements of on-site inspection for protection equipment in intelligent substation, combined with spatial anchor, an AR inspection system with man in the loop and virtual reality integration is proposed based on the augmented reality technology. The overall design is carried out from multiple dimensions such as system architecture, business functions, business processes and network topology, and the technical methods and key technology implementation methods such as target recognition, spatial positioning, and near-eye display are elaborated in detail, the implementation of inspection task is verified by field application from macro and micro scales. The system realizes the enhanced display of real equipment in physical space and various equipment status, monitoring data, operation and maintenance information in virtual space, assists personnel to carry out inspection activities such as inspection route navigation, equipment location, inspection operation and process record, improves the patrol quality, efficiency and upgrade methods, it has important application value.
\end{abstract}

\section{Introduction}

Equipment inspection is an important content of substation operation and maintenance management[1].Regular and irregular inspections, such as routine inspections, comprehensive inspections, and special inspections, are used to accurately grasp the operation of equipment, find equipment abnormalities or defects in time, and eliminate hidden dangers. It is an important measure to ensure the safe and reliable operation of substation equipment and power grid.

On the one hand, with the construction of intelligent substations, remote monitoring of the operation status of the power grid can be partially achieved through equipment operation monitoring networks, inspection robots and other means, but on-site inspections are still essential.On the other hand, with the development of augmented reality (AR) technology, using location registration, enhanced display and human-computer interaction and other technical means to match and superimpose virtual pictures or information into real scenes through AR glasses to assist on-site inspectors to obtain all necessary information in time, can effectively improve the quality and efficiency of on-site inspection.

At present, the online monitoring and intelligent diagnosis system of intelligent substation protection equipment realizes the management of equipment through the SCD (Substation Configuration Description) file, and obtains the information of merge unit, intelligent terminal, protection device, safety automatic device and switch through the station control layer network and process layer network, realizes the operation monitoring, intelligent diagnosis and fault analysis of the protection equipment, but the application mode of the computer terminal can't meet the ability to patrol the scene anytime and anywhere.

According to the Technical Specification for On-line Monitoring and Diagnosis Devices of Protection Equipment in Intelligent Substation of State Grid Corporation of China,this paper takes the on-site inspection of protection equipment in intelligent substation as an example, based on the on-line monitoring and intelligent diagnosis system of protection equipment, designed and developed the AR inspection system for protection equipment of intelligent substation with human-in-the-loop, virtual and real integration, and WYSIWYG, and conducted a demonstration application.

\section{System objectives}

For the on-site inspection task of the protection equipment of the substation, the AR inspection system uses the online monitoring and the intelligent diagnostic system of the protection equipment as the data support, completes the inspection task and data acquisition through the computer terminal, and combines with AR glasses and on-site anchors for visual guidance, process recording and objective evaluation of inspections. The system supports

*liuyumin16@mails.tsinghua.edu.cn 
gestures, voice and other human-computer interaction methods, assisting inspectors to obtain various information such as electrical diagrams, parameter information, operating status, alarm information, status evaluation, etc. of the equipment as needed, and display the augmented information on the real equipment, directly feed back the data and diagnosis results, So the inspectors can comprehensively, quickly and efficiently troubleshoot and analyze the problem on the site, solve the problems of inconvenient information acquisition, weak task guidance and unobjective evaluation in traditional inspections, improve the quality and efficiency of inspections.

\section{Overall design}

\subsection{System architecture}

Under the unified standard specification and security system of the State Grid, according to the processing level of data information flow, the structure level of the AR inspection system can be divided into equipment sensing resource layer, network transmission layer, data model resource layer, platform tool layer and business application layer, as shown in Figure 1.

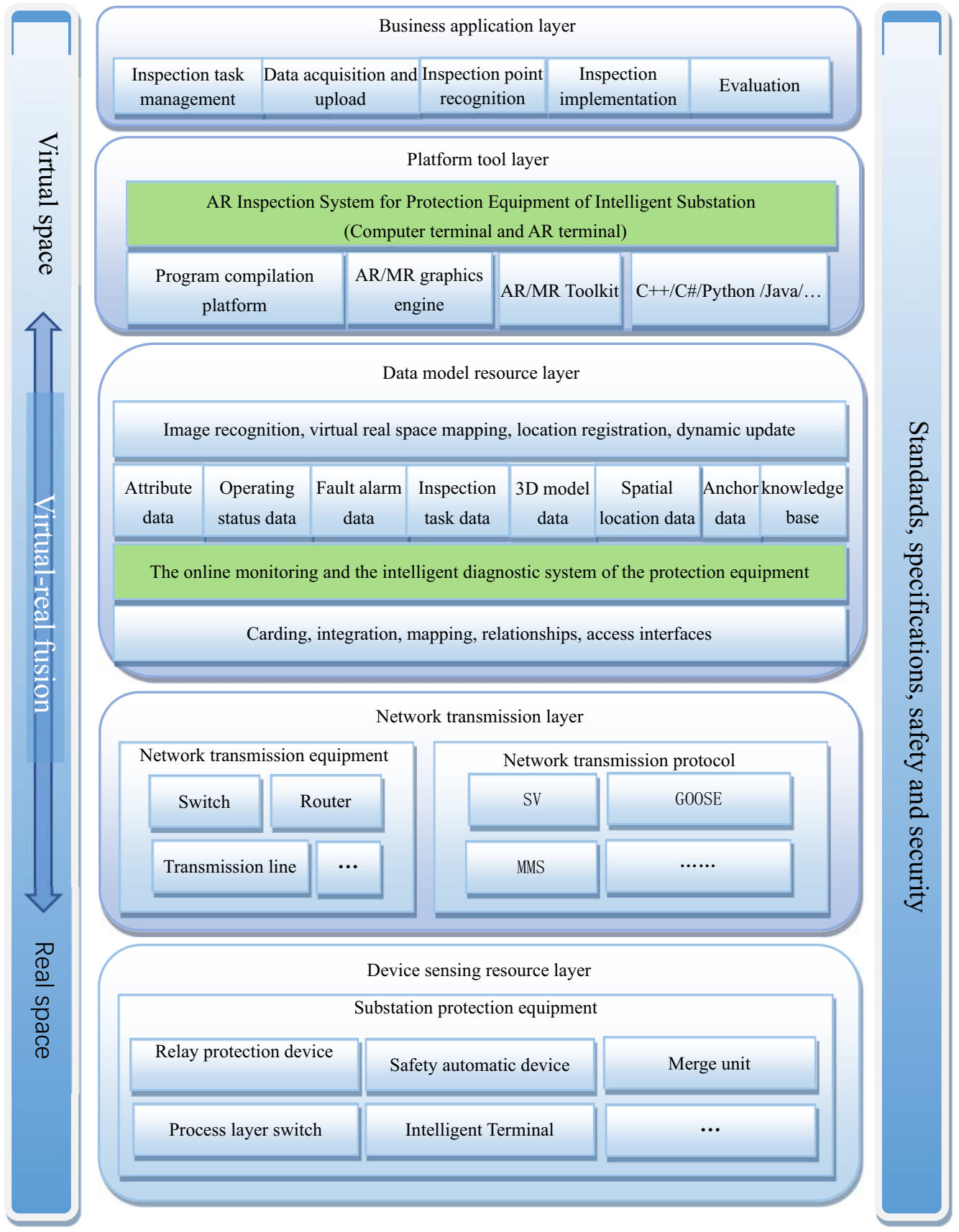

Figure 1. System architecture diagram

The system collects monitoring data from protection equipment through network equipment and network transmission protocols, obtains the operating status and diagnostic evaluation data of substation protection equipment based on the online monitoring and intelligent diagnostic system, and classifies them by business 
applications, form a data set that supports system business applications by combining with inspection tasks and onsite anchor identification , model, knowledge base and other data.

Based on image recognition, virtual and real space mapping, location registration and dynamic update algorithm models, under the unified technical framework for computer terminal and AR terminal, provide specific business application functions such as inspection task management, data acquisition and upload, inspection point identification, inspection task implementation and evaluation, and ultimately form the matching mapping and data fusion of substation physical space and virtual space.

\subsection{Functions}

The AR inspection system includes two complementary logic application forms, the computer terminal and the AR terminal, and is used in conjunction with the identification anchors distributed at the inspection site. Its functional design is shown in Table 1.

Table 1. AR inspection system functional list

\begin{tabular}{|c|c|c|c|c|c|}
\hline \multicolumn{2}{|r|}{ Computer terminal } & \multicolumn{4}{|c|}{ AR terminal } \\
\hline (1) & Inspection task & (1) & Inspection point recognition & - & Route record \\
\hline \multicolumn{2}{|c|}{ management } & (2) & Inspection task & - & Image recording \\
\hline$\diamond$ & Task planning & \multicolumn{2}{|c|}{ implementation } & - & Audio and video recording \\
\hline$\diamond$ & Task list & $\diamond$ & Route navigation & (3) & Inspection task evaluation \\
\hline$\diamond$ & State management & $\diamond$ & Device positioning & $\diamond$ & Inspection process \\
\hline$\diamond$ & Task query & $\diamond$ & Equipment inspection & & evaluation \\
\hline (2) & Inspection data & - & Optical fiber physical link & - & Visualization of Inspection \\
\hline acquisit & & & monitoring & & routes \\
\hline$\diamond$ & Data interface & - & Secondary circuit monitoring & - & Time-consuming statistics \\
\hline$\diamond$ & Data acquisition & - & Interval status information & & of Inspection points \\
\hline- & Task data & & monitoring & $\diamond$ & Evaluation of inspection \\
\hline- & Operating data & - & Historical information query & & results \\
\hline- & Attribute data & - & Fault location & - & Task completion rate \\
\hline- & Alarm data & - & Diagnostic analysis and early & - & Task compliance rate \\
\hline- & Other data & & warning & - & Overview \\
\hline$\diamond$ & Data distribution & $\diamond$ & Inspection record & $\diamond$ & Inspection data upload \\
\hline
\end{tabular}

The computer terminal obtains various monitoring and evaluation data from the online monitoring and intelligent diagnosis system of the protection equipment through the data interface, dynamically extracts the data according to the inspection task, and downloads it to the AR terminal. It mainly implements planning management functions such as inspection task planning, task list, task status, and task query, as well as data extraction functions such as data interface and various data acquisition and distribution.

The AR terminal is deployed on the AR glasses. After identifying and positioning the inspection anchor points with image recognition, it assists the inspection tasks such as route navigation, device positioning, auxiliary operations and process recording, and provides online monitoring and intelligent diagnosis of protection equipment.

The online monitoring of protection mainly includes optical fiber physical link monitoring, secondary loop monitoring, interval status information monitoring and historical information query functions, and visually realizes the connection and disconnection of optical fiber physical links, optical fiber signal loops and status, pressure plate status, protection actions and alarms Information etc. Intelligent diagnosis mainly realizes fiber link fault location, protection equipment abnormality/alarm board location, primary and secondary operation consistency check and diagnosis warning. After the inspection is over, the evaluation and data of the current task are uploaded to the computer for storage.

\subsection{Business process}

According to the implementation process of inspection tasks, the use of AR inspection system can be divided into three stages: inspection preparation, implementation and evaluation. Its business process design is shown in Table 2 .

Table 2. AR inspection system business process

\begin{tabular}{l|cl}
\hline \multicolumn{1}{c}{ Inspection stage } & \multicolumn{3}{c}{ Table 2. AR inspection system business process } \\
\hline & $(1)$ & Start the inspection system (computer terminal and AR terminal); \\
& $(2)$ & Enter the computer terminal of the system; \\
inspection & $(3)$ & Obtain inspection tasks; \\
preparation & $(4)$ & Obtain inspection data; \\
& $(5)$ & Download the data to the AR terminal of the inspection system; \\
& $(6)$ & Wear AR glasses and start patrol. \\
\hline
\end{tabular}




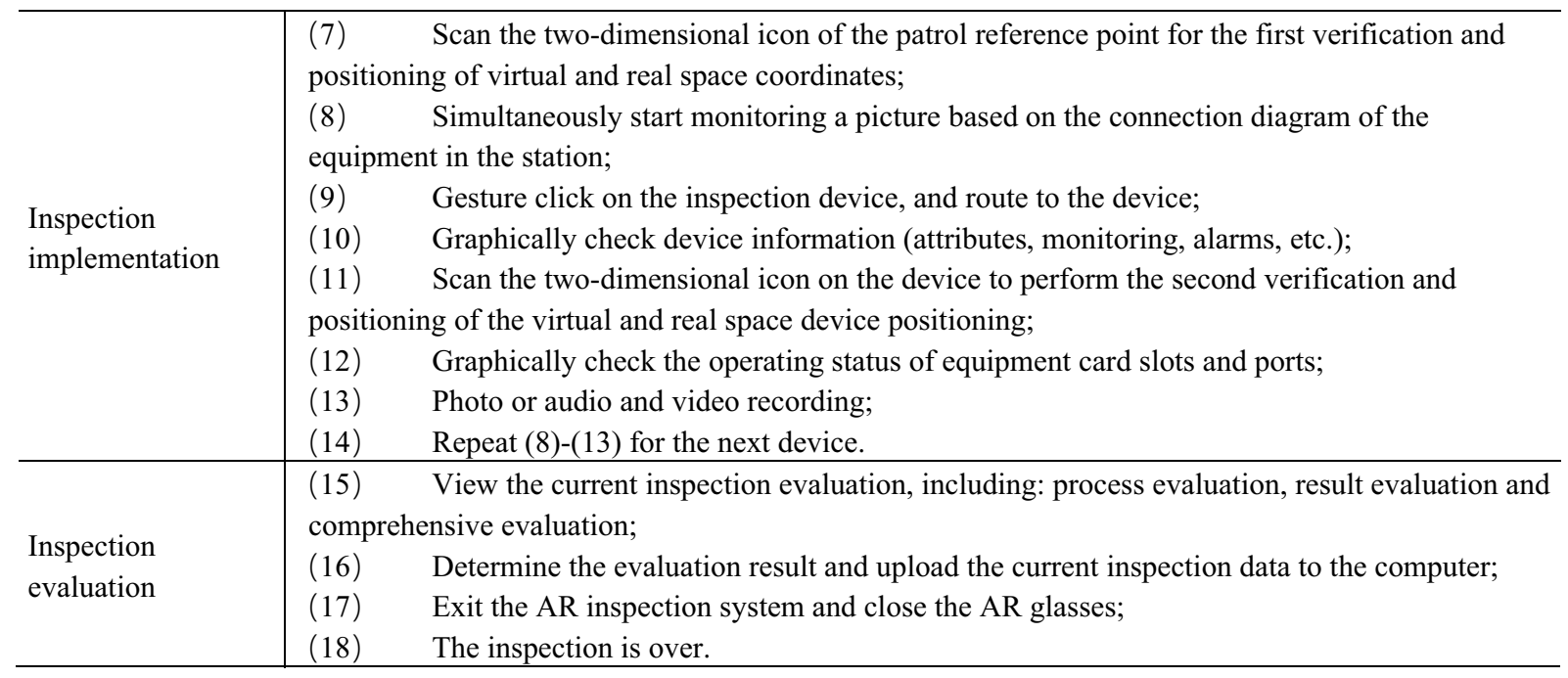

The inspection preparation stage mainly completes the system startup, obtains tasks and extracts data from the computer terminal, and downloads to the AR terminal. In the implementation stage of the inspection, based on AR terminal location registration, complete the assistance and support of the on-site inspection operations, mainly complete the reference anchor identification, coordinate origin positioning, inspection route navigation, inspection equipment identification positioning, information push and enhanced display, auxiliary operations, process records, verification of virtual and real spaces, etc.. The inspection evaluation stage, the implementation process of the current inspection task is evaluated according to specific rules, and the inspection implementation data is uploaded to the computer for storage.

\subsection{Network topology}

According to the safety regulations and requirements of the substation, the online monitoring and intelligent diagnosis system of protection equipment realizes the collection and intelligent diagnosis of equipment monitoring data through network protocols such as SV, GOOSE, and MMS. The computer terminal of the AR inspection system reads the monitoring and diagnostic data from the online monitoring and intelligent diagnosis system of the protection device through the network communication unidirectional isolation device from the proxy communication server, and downloads the data offline to the AR terminal of the inspection system through the data line. The AR terminal completes the location registration by performing image recognition on the anchor marks distributed on the site, so that information such as videos, images, or text is superimposed and displayed on the real device. The network topology is shown in Figure 2.
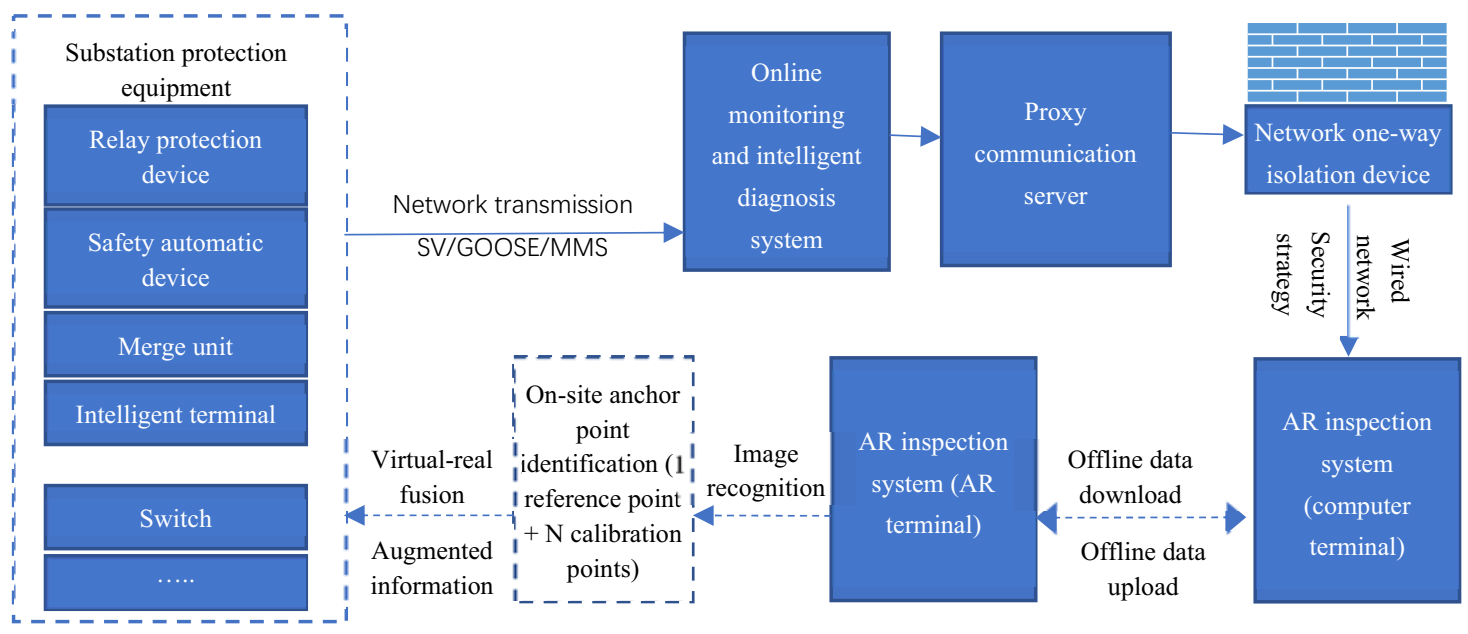

Figure 2. AR inspection system network topology 


\section{Technical approach}

The realization of AR inspection system mainly includes three aspects: equipment modeling, field anchor point and software development.

Equipment modeling mainly uses three-dimensional modeling tools to implement geometric model construction, texture and material mapping of objects such as inspection equipment combinations, cables, terminals, etc., providing realistic three-dimensional object simulation display for the inspection system

On-site anchors mainly implement location registration, equipment identification and spatial positioning functions, and are distributed in different locations of substations according to inspection requirements. These anchors adopt uniform size, specification and elements, and use a two-dimensional image with different graphic feature information to represent 1 reference point and $\mathrm{N}$ calibration points respectively. The reference point is the coordinate origin of the inspection, and the check points undertakes the dual functions of coordinate calibration and equipment identification, the amount of calibration anchors is positively related to the size of the physical space and the number of equipments.

Software development is the key to realize the function of AR patrol system, which includes two application forms: computer terminal and AR terminal. Under the unified standard specification, the computer terminal uses $\mathrm{B} / \mathrm{S}$ architecture to develop pages with JDK, the AR terminal uses Microsoft HoloLens head-mounted display device, and on the Windows 10 platform, uses Microsoft Visual Studio 2015, HoloLens emulator, Unity2018, Mixed Reality Toolkit 2.0 (MRTK 2.0) and other software for development.

\section{Key technology}

The AR inspection system follows the existing inspection specifications and operating procedures, which is the improvement of traditional on-site inspection methods. It needs to solve the problems of accurate and fast recognition of inspection equipment, location tracking and augmented information display. It involves key technologies such as target recognition, spatial positioning and near eye display.

\subsection{Target recognition [3]}

The key factors that affect the practicality of the AR inspection system are recognition accuracy and recognition efficiency. For the application scenario of substation inspection, the selection of target recognition method mainly considers two factors. The first factor is the characteristics of the object. The shape characteristics of the substation protection equipment and its control cabinet are unified and similar, the accuracy of directly identifying the equipment is not high enough to meet the actual application. The second factor is the actual requirements for spatial positioning and standardization of inspection sites.

Therefore, under the unified standard specification, a two-dimensional image containing the corporate logo and the device information is designed, and the image storage and equipment corresponding relationship are realized in advance. When patrolling, you can accurately complete the device identification and position verification by identifying the two-dimensional code image posted near the device. The experiment proves that, unlike the direct recognition equipment, the recognition error using the two-dimensional image recognition will not occur, the recognition accuracy can reach $100 \%$, and the recognition efficiency has also been greatly improved.

\subsection{Spatial positioning [4]}

Spatial positioning is mainly used to track the indoor location of inspectors and register the location of inspection equipment. Under the unified coordinate system and measurement standards, the physical space and virtual space are mapped at a 1:1 real-world scale, realizing the process records of inspectors and the display of augmented information on real equipment, which provides the basis for inspection process evaluation and inspection information display.

In the aspect of patrol location tracking, considering the system's universality, practicability, and particularity of the application scenario, the Hololens inside-out tracking technology is adopted, and the method of "Spatial Anchor [2] dynamic positioning method with one reference point and multiple calibration points" is used to realize the passive tracking of the indoor location of personnel. The specific implementation method is as follows:

(1) Arrange a reference point and multiple calibration points in the physical space, and take the reference point as the reference origin, measure and mark the relative coordinate position of each point in the physical space in advance.

(2) Construct the absolute coordinate system of the virtual space, identify the reference point mark through Hololens, and complete the definition and registration of the origin of the absolute coordinates of the virtual and real space.

(3) Use Hololens' small-area space inertial navigation capability to realize the determination and dynamic update of personnel position and movement direction.

(4) Use Hololens to identify multiple calibration points distributed in the scene, complete the calibration and dynamic correction of the virtual-real space positions.

The position registration of the inspection equipment is dynamically updated using a relative coordinate system. Unlike the absolute coordinate system used for position tracking throughout the entire inspection process, the relative coordinate system is only applicable to the current equipment.

The four-step method of "measurement, modeling, identification, registration" is used to realize the augmented display, as follows: 
(1) Measurement: measure and obtain the relative spatial position and layout relationship between the calibration point and each component unit or component of the equipment in the physical space.

(2) Modeling: Use the calibration point as the reference origin to build a $3 \mathrm{D}$ scene of the device in the virtual space, which mainly includes the virtual modeling of the component units or components of the device, the display position and method of various information defined in advance.

(3) Recognition: Use Hololens to identify the calibration point label attached to the inspection equipment, obtain the current coordinate position and equipment name, and dynamically create a relative coordinate system with the label point as the origin.

(4) Registration: In the relative coordinate system, the position of the virtual space and the physical space is registered to to realize the enhanced display of virtual information on the real device.

\subsection{Near-eye display [5]}

AR technology belongs to the category of near-eye display, and user comfort is crucial. The factors that affect comfort mainly include hardware weight, display field of view, human-computer interaction, holographic image display distance, image clarity and update frequency. When the AR hardware is selected, the improvement of near eye display is mainly from the aspects of human-computer interaction, holographic image display etc. In the aspect of human-computer interaction, considering the characteristics of application scene and interaction content, one or more technical means such as gestures, speech, and gaze can be used to achieve natural interaction with virtual space; in the aspect of holographic image display, it mainly optimizes the display area, frame rate and clarity.

In terms of display area optimization, the discomfort caused by "visual convergence-adjustment conflict" should be avoided, because with the decrease of visual distance, the discomfort caused by "visual convergenceadjustment conflict" will increase exponentially. It is recommended that the best placement area of the hologram is 1.25 meters to 5 meters. It is not recommended to render the hologram within 40 centimeters, and place the rendering clipping plane at 30 centimeters, while avoiding the user's fast switching

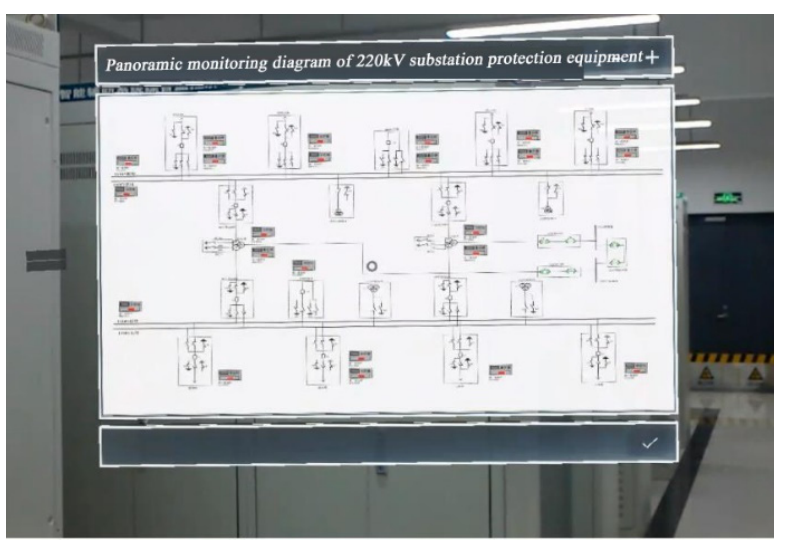

Figure 3. Panoramic monitoring diagram between near focus and far focus.Taking horizontal observation as an example, it is recommended that the best line-of-sight angle is 10-20 degrees horizontally downward, avoid vertical movement of the line of sight more than 10 degrees upward and more than 60 degrees downward, and avoid horizontal rotation angle of more than 45 degrees.

In terms of frame rate, it is suggested that the lowest frame rate is 60 FPS for holographic image rendering, which can reduce the visual jitter and the overall delay, avoid the jitter of holographic image in motion, reduce the prediction error and improve the stability of holographic image.

In terms of clarity, holographic images are displayed in high-resolution or ultra-high-definition resolutions, especially the text. Text clarity depends on various display attributes (such as pixel density, brightness, contrast), lens attributes (such as chromatic aberration), and text/font attributes (such as thickness, spacing, trim, and other specific font characteristics, as well as font color, background colour). It has been proved that for the display content presented at a distance of 2 meters from the user, the least clear font size (the measurement size from the font baseline to the rising stroke) is about $0.35^{\circ}$, while the comfortable font size is at least about $0.5^{\circ}$.

\section{Application verification}

The AR inspection system realizes the seamless overlay display of the status, monitoring data, operation and maintenance information of various devices in the physical space and the virtual space. It has the characteristics of mobile, digital and intelligent, and provides functions such as online monitoring and intelligent diagnosis of protection equipment. It provides technical means for the implementation of inspection tasks from the macro and micro scales. Some field application effects are shown in Figure 3-Figure 7 shown.

Figures 3 and 4 are the connection diagram of the substation protection equipment and the overview diagram of equipment status monitoring of the whole station based on the SCD file, which together constitute the "macro diagram" of the station equipment monitoring, and visualize the operation status and link relationship of equipment of the whole station from the global perspective.

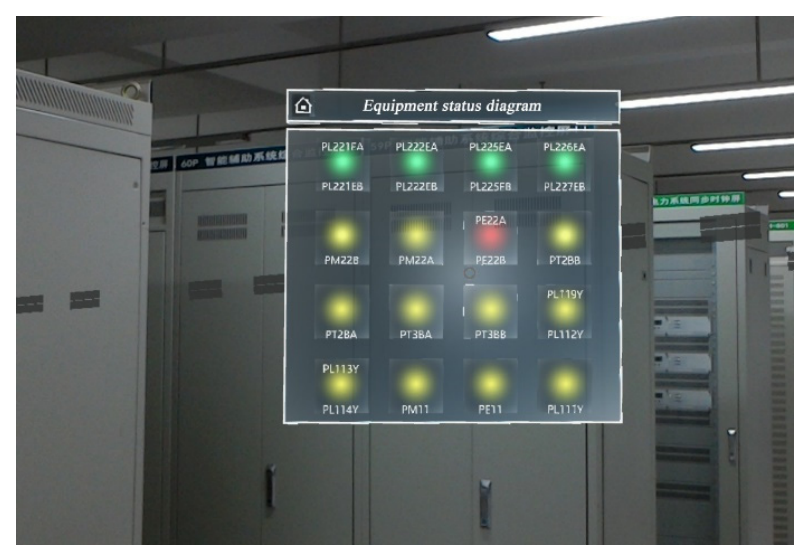

Figure 4. Equipment status diagram 
Figure 5-Figure 7 are respectively the superposition of operation status, monitoring data, fault alarm and other information on the real equipment, and the display in combination of text, chart, 3D model, etc., to form a "micro diagram" for monitoring and fault diagnosis of a patrol equipment, and the visual monitoring of the operation status of the equipment from a local perspective.

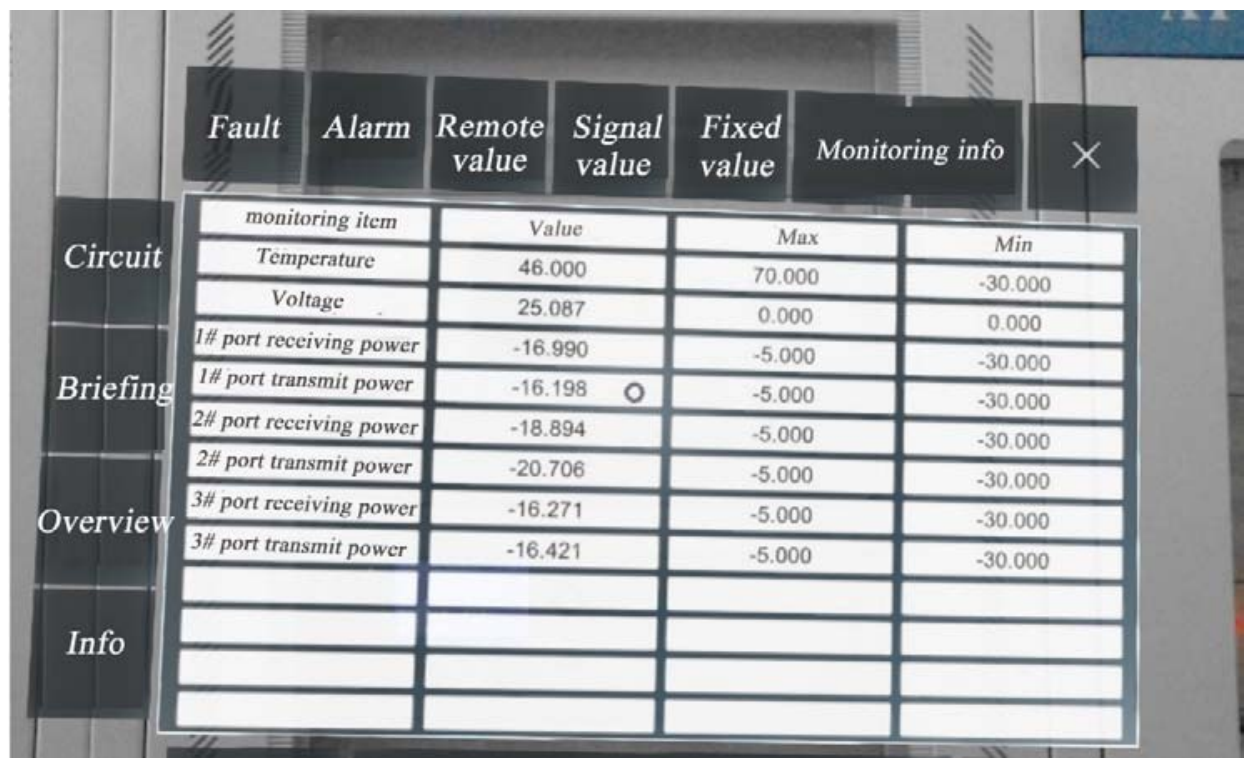

Figure 5. Monitoring information panel

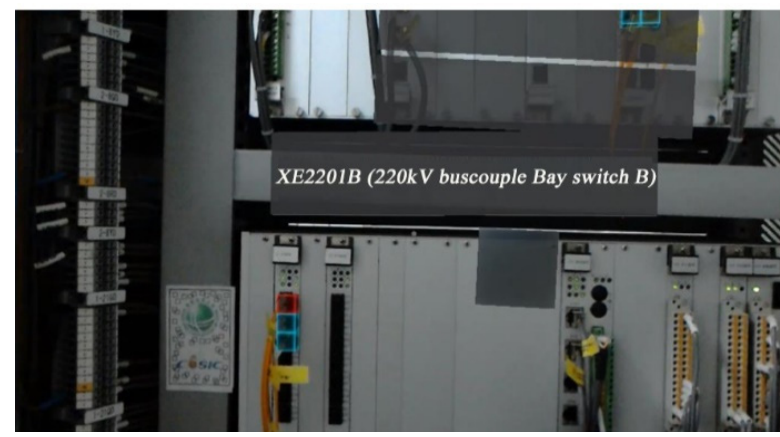

Figure 6. 3D geometric model and information for augmented display

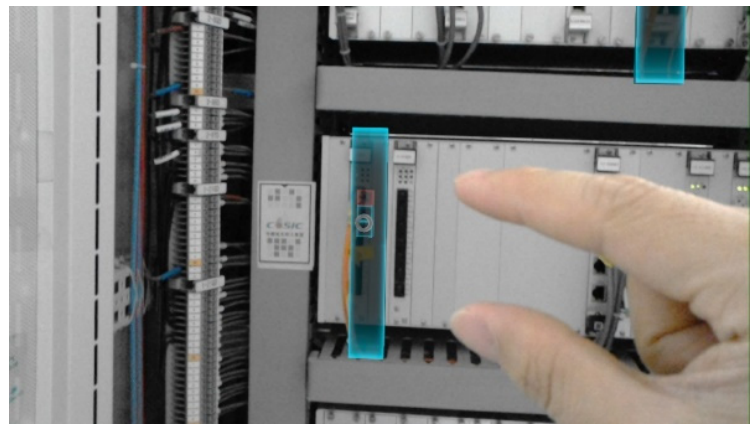

Figure 7. Augmented display of optical port connection status

\section{Conclusion}

Augmented reality technology has important application value in smart grid construction and maintenance [6]. Intelligent substation is an important part of smart grid construction, and protection equipment is the foundation and key to maintaining safe, reliable, and stable operation of substations. According to the requirements of on-site inspection of intelligent substation protection equipment, this paper introduces an AR inspection system with man in the loop, virtual reality integration and WYSIWYG, which is based on the data of online monitoring and intelligent diagnosis system of protection equipment. The implementation of inspection tasks such as navigation, equipment positioning, inspection operations and process records will effectively improve the operation safety, operation capacity and operation efficiency of power operation and maintenance, and realize the transformation of inspection from traditional "passive", "rough", "empirical" to "active", "lean" and "intelligent".

\section{References}

1. Wang Hailin. (2018) Analysis on the inspection work of substation equipment. Science and Information, 33:93-93.

2. Lei Zhenjiang, Wang Lei, Cui Jisheng, Chen Shuo, $\mathrm{Xu}$ Zhengqing, Yu Hailong, Meng Hao, Liu Guozhong. (2019) A Substation Simulation Training System Based on HoloLens. Computer and Modernization, 11: 94-99.

3. Huang Ang, Shen Yi, Tang Yuanyuan. (2019) Operation and Maintenance Design of Industrial Equipment Based on Mixed Reality Technology. Ordnance Industry Automation, 38(9): 72-77.

4. LIU Zijun, JIAN Xuezhi, SHI Bonian, ZHANG 
Huixian. (2019) Operation and Maintenance Simulation for Smart Substation Equipment Based on Augmented Reality Technology. Southern Power System Technology,13(11):69-75.

5. Microsoft. (2019) HoloLens Development documents. https://docs.microsoft.com/zh-cn/hololens/.

6. Xu Han, Yang Baolong, Yang Yufeng, Congli, Yang Yu, Qiang Sheng. (2019) Application of augmented reality technology in operation and maintenance of power communication network. In: Research and Application of Power Communication Technology, Beijing, pp.413-416. 